\title{
Advanced Pervasive Clinical Decision Support for the Intensive Care Unit
}

\author{
Femke De Backere, Filip De Turck \\ Ghent University - IBBT, \\ Department of Information Technology (INTEC) \\ Gaston Crommenlaan 8, bus 201, \\ 9050 Ghent, Belgium \\ Email: femke.debackere@intec.ugent.be
}

\author{
Kirsten Colpaert, Johan Decruyenaere \\ Ghent University Hospital, \\ Department of Intensive Care \\ De Pintelaan 185, \\ 9000 Ghent, Belgium \\ Email: kirsten.colpaert@ugent.be
}

\begin{abstract}
Clinical decision support systems (CDSS) already have proven their use in supporting physicians and nurses in their daily responsibilities. However the uptake of these systems remains low. This is due to a number of reasons, such as little to no integration within the workflow of the medical staff, a multitude of available devices and a large amount of medical data that has to be processed. This research focusses on closing the communication gap between software engineers and domain experts and on integrating CDSS into the workflow of the medical staff by providing support at the right time, at the right place on the most suitable device, based on the current context. This way the variation in care can be reduced and costs are minimized.
\end{abstract}

\section{BACKGROUND TO THE RESEARCH}

As the complexity and size of medical information and data keeps rising, the need for clinical decision support systems (CDSS) grows at an ever increasing pace. CDSS are computerdriven technology solutions, developed to provide support to physicians, nurses and patients using medical knowledge and patient-specific information. Thus, these systems will not replace the medical staff, but will merely give advice and guidance. This way, they are able to take all relevant data and information into account. Examples of such data are information from databases or the electronic health record (EHR) [1]. By filtering the information in an intelligent manner and presenting it to the medical staff at the appropriate moment, these systems can improve health care [2]. CDSS can be used in every aspect of the care process, from preventive care and diagnosis to monitoring and follow up. Studies have already shown that these systems improve quality, safety and effectiveness of medical decisions, resulting in improved patient care, higher performance of the medical staff and more effective clinical services [3]. Nevertheless, the uptake of CDSS is rather low and this is due to a number of factors [4].

First, one of the main problems in the use of CDSS is the integration of applications into the current workflow of the medical staff [5]. Kawamoto et al [6] concluded that CDSS are more successful when integrated into the work process of the medical staff. This also means the integration with existing information systems and devices in the hospital. In a computerized Intensive Care Unit (ICU), a computer is located next to every bed. In addition, each department also has a unit PC and physicians usually have a personal desktop and telephone. An overview of this situation is shown in Figure 1. Moreover, the number of devices on an ICU is steadily increasing in recent years [7]. Currently, these devices are not optimal embedded within CDSS. Sharing information at the right time and place has a large influence on the use of these systems and on the performance of the medical staff, moreover it is time-saving [8].

Second, next to offering information to the users at the right time and place, the context of the user is also an interesting point of focus. Context-aware applications can take technologies, such as wireless technologies, sensors, mobile tools and handheld computers or smartphones into account. This can support health care providers in managing their tasks and will at the same time increase the quality of care [9]. In particular, ICUs sometimes contain complex and difficult situations and pose an interesting challenge for these systems.

The third problem is representing all the relevant information of a specific patient. In the ICU, up to 200,000 parameters are collected for each patient on a daily basis [10]. These parameters are mainly originating from examinations and from monitoring data. Visualizing this data in an optimal way and selecting only the most relevant information is a challenging task [11]. It is also important to build new knowledge from the gathered data [12].

Last, CDSS are often based on clinical guidelines. Clinical guidelines are systematically developed documents used in hospital settings to standardize and structure treatment or diagnostic processes [13]. A typical guideline departs from observations, employs evidence-based practices and hence leads to conclusions and recommendations. If necessary, it can be deviated from, based on specific hospital policies or expert knowledge. Thus, guidelines are an indispensable tool in order to guarantee a high quality of care, while restricting redundant expenses [3]. Currently however, they are largely handwritten, text-based or depicted as flowcharts. The translation of written documents into working applications is very time-consuming and requires software developers to be thoroughly familiar with the medical problem domain. It requires a strong cooperation between domain experts and software developers, which is complicated by the communication gap between both worlds [14]. Even more important, multiple representation languages 


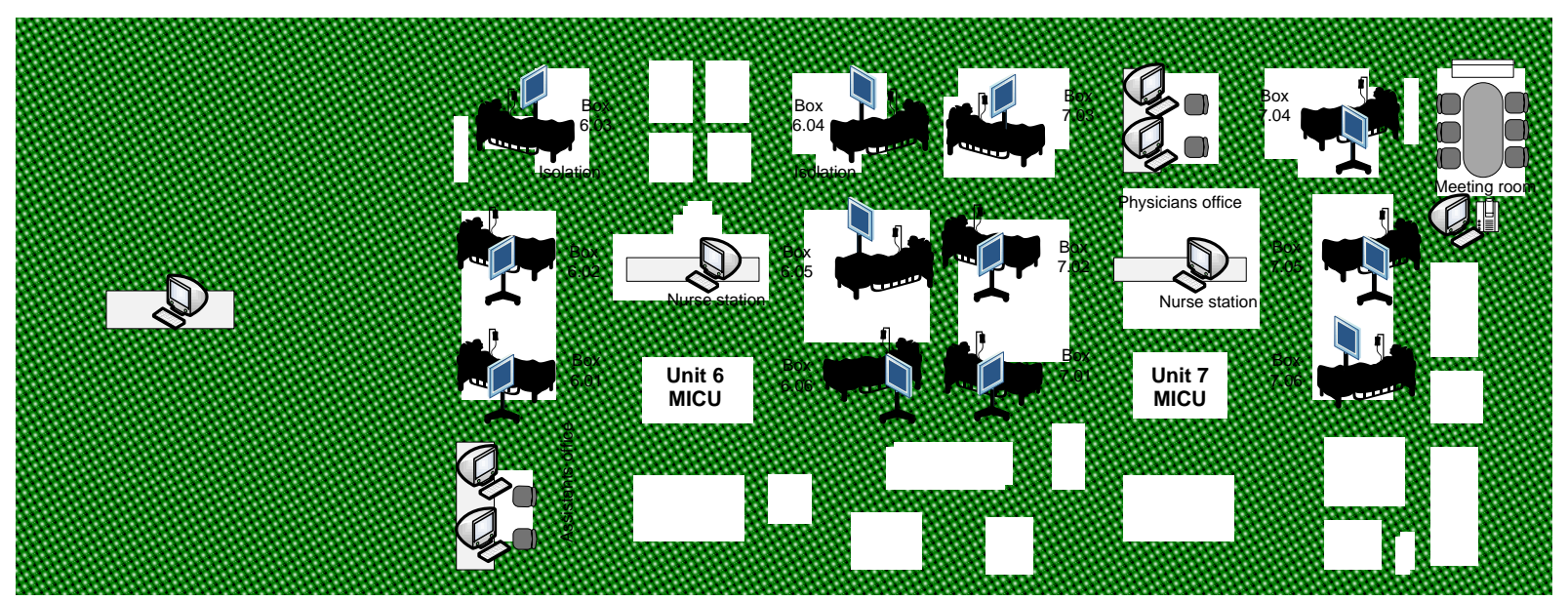

Fig. 1. Devices in the ICU

are available, each with their own advantages, specifications and goals. It is difficult to decide which format is the most appropriate in a given situation [15].

The remainder of the article is structured as follows. In Section II the specific problem the research will address is presented. Section III is devoted to the methodological approach and Section IV describes the work done to date. Section V will discuss the planned work, while Section VI describes the contribution of this research. Finally, in Section VII, specific issues for the doctoral consortium are addressed.

\section{SPECIFIC PROBLEMS THE RESEARCH WILL ADDRESS}

As already mentioned in the previous section, the uptake of CDSS remains low. To this end, my $\mathrm{PhD}$ focusses on finding solutions for the integration of CDSS in the workflow of the medical staff of the ICU. The research questions are divided into 3 topics, each addressing a specific issue. They are formulated as follows:

- How can clinical guidelines be translated into working computer programs? Is there a way to close the communication gap between domain experts and software developers?

- With the ever growing presence of various devices and computers in the ICU, is it possible to use the most appropriate device, depending on the circumstances and seamlessly switch to another device when it suits the user most? Is it realistic to take preferences of members of the medical staff into account? How can the large amount of available data be optimally visualized on the devices and computers? Is it possible to select the data in an efficient way?

- How can knowledge, captured by the CDSS, contribute to a more improved system? Can we learn from the behavior of the users and the use of the system in general and optimize it, based on the acquired information?

\section{Methodological APPROACH}

To address these problems, the research is conducted in three different stages.
First, an architecture will be developed, capable of plugging in different modules, enabling an extensible platform. The main focus is this part of the research is the automatic translation and deployment of clinical guidelines into working applications. These applications can easily be plugged into the platform. By doing the translation of clinical guidelines automatically, the communication gap between domain experts and software developers can be closed. Another emphasis of the decision support platform, in this phase, is taking into account aspects with respect to human-computer interaction.

Second, the platform will be extended with algorithms to provide decision support at the right time and place, based on the current context of the medical staff. These algorithms will be responsible for selecting the most appropriate device based on the circumstances, the location and the preferences of the end users. For example, a physician is using his smartphone to visualize information about a specific patient, but he is in close proximity of his desktop computer screen. Then, it could be more appropriate to visualize the data on the larger screen, as data can be shown in a more detailed manner.

Last, it is also important for the platform to learn from actions taken and choices made by the medical staff in the past. This way, the devices will always be integrated into the workflow of the staff, also when behavior or preferences change. Therefore, self learning algorithms will be developed.

After each of these phases, a prototype will be implemented. In addition, the design will be evaluated based of simulations and tests. Iterative optimizations can be carried out in order to improve algorithms and techniques. As this research is done in close cooperation with the Department of Intensive Care of the University Hospital Ghent, specific use cases will be chosen to test the platform.

\section{WORK DONE TO DATE}

The first 2 years of the doctoral research focussed on developing a methodology and implementing a proof of concept to facilitate the translation of clinical guidelines into working applications.

Based on the architectural drivers modifiability and extensibility, an architecture was developed to satisfy all requirements 


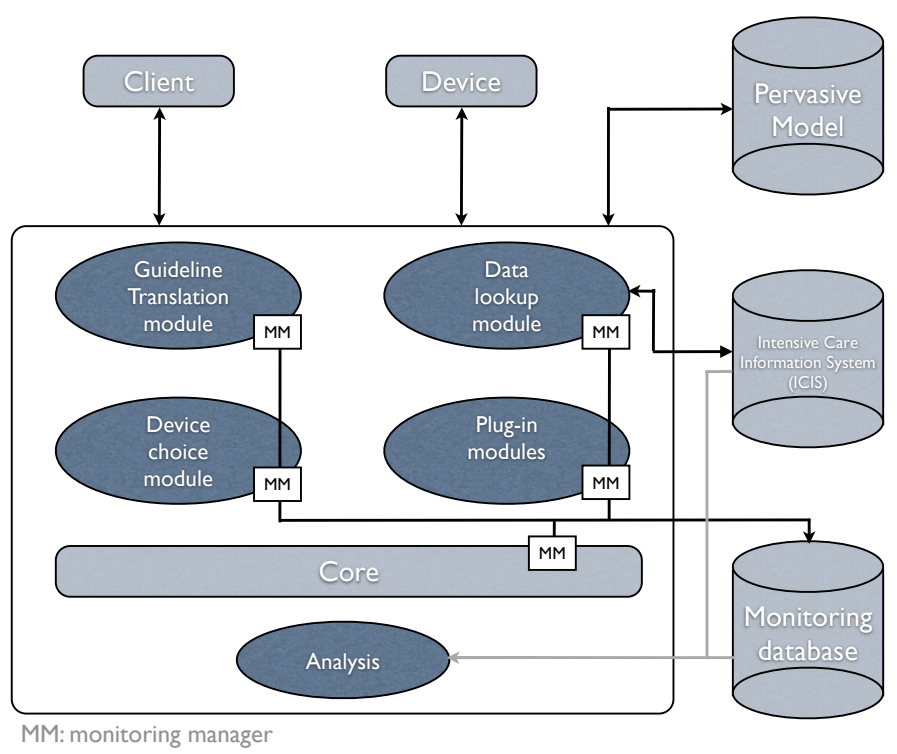

Fig. 2. Overview of the high level architecture of the pervasive clinical decision support system

and address the problems mentioned in Section II. The framework is based on the microkernel pattern [16]. This pattern is mainly designed for systems in changing environments, which have to be extremely adaptable and extensible. To achieve this, a minimal, functional core is used, which is split off from the rest of the system. This kernel works together with other components, which can easily be replaced or modified. The major advantage of using a microkernel is the increased reliability of the engine and its extensibility. The high level overview of the pervasive framework is depicted in Figure 2.

The architecture consists of six modules. Each module can be extended with a monitor managers (MM), to capture useful information in a non-intrusive way. Stated differently, we do not want logging code to be tangled with the actual functionality, nor do we want to influence the execution times of our framework beyond a minimal threshold. The logging data will be captured into the Monitoring database.

The Core module is responsible for handling all high level functionality and delegating requests to the responsible module. The Data lookup module (DLM) will communicate with the Intensive Care Information System (ICIS) and will query this database or other databases residing in the ICU environment, such as the Picture Archiving and Communication System (PACS) and the GLIMS-Lab information system. This module is monitored and in case of low performance, the load can be spread on different DLMs.

Next, the Guideline Translation module was developed. This phase was preceded with an in-depth (literature) study into clinical practice guidelines (CPG) and computer-interpretable guidelines (CIG). Nowadays, clinical guidelines are often represented as plain text, which makes them difficult to handle. Often, these guidelines are handwritten or represented as flowcharts. Moreover, it becomes more and more complex to teach such guidelines to physicians. This also makes it inconvenient to search and execute the proper guideline.
Therefore, research was done into ways to translate flowcharts into executable programs.

In a first phase, research was done towards the representation format of guidelines. Therefore, the sedation guideline, used for the depression of consciousness of critically ill patients as applied in the ICU of the University Hospital Ghent, was analyzed. These guidelines consist of 5 straightforward flowcharts. These flowcharts were then manually translated into the Unified Modeling Language (UML) [17], in order to compare the differences and similarities ${ }^{1}$. Drools Rule Flow, nowadays called jBPM $5^{2}$, was chosen as a technology to represent these guidelines. This technology has a close resemblance with the original format of the clinical guidelines and it can easily be visualized to the medical staff [18].

After further research into UML and how it can be used in conjunction with CPGs to resolve known problems with the translation of guideline formats into working computer applications, UML was chosen as an internal format because of its comprehensibility and interpretability. To this end, an execution and translation module was developed, which is capable of executing UML-based guidelines and mapping existing guideline formats, such as GLIF3, on this new format. By using UML as an intermediate format on the one hand and by using the microkernel pattern on the other, various CPG formats can be executed on one engine. This reduces development and implementation cost as only a new format mapping has to be added to the execution engine. After the translation process, optimization algorithms can be executed in order to improve the guidelines even further. After an initial evaluation, we can conclude that the execution of the guideline is very efficient. The overhead is mainly caused by the dynamic loading of the services, used in the diagrams. The framework accelerates the translation and development of medical guidelines to model patient care and reduces the communication gap between domain experts and software developers [19].

During this research phase, several use cases were used: the Modified Schofield flowchart to calculate the calorie need for burn patients [19], the antibiotic dosage guideline to calculate the dose and type of antibiotics given to the ICU patients [20] and the sedation guideline [18].

In a later stage of the research, other Plug-in modules can be easily added to the framework. The other components of the framework will be addressed in the Section V.

\section{PLANNED WORK}

The next phase of the research will focus on developing contex-aware pervasive algorithms for the Device choice mod$u l e$, in order to perform decision support at the right time and place, based on the context of the user. This module is responsible for showing the information on the most appropriate device and if another, more suitable, device becomes available, switch fluently.

\footnotetext{
${ }^{1} \mathrm{http}: / /$ www.biomedcentral.com/content/supplementary/1472-6947-10-3S3.ZIP

${ }^{2}$ http://www.jboss.org/drools/drools-flow
} 
At first, a Pervasive model will be developed. This is an ontology, capturing all the knowledge about the ICU domain, as well as the available devices in the ICU and the characteristics of the medical staff. The ontology has some core concepts, such as Device, Person and Location. This model also consist of domain-specific rules. Further research has to be done into rule languages and reasoners, in order to choose the best combination. As performance is certainly one aspect of the framework, the selection of a specific device and switching to another should happen in a certain timeframe. Application-specific rules and also use cases will be developed in close cooperation with the ICU department. Based on these use cases, a proof of concept can be created.

Second, data mining algorithms will be used to optimize the use of devices in the ICU. The algorithms will use the data captured by the Monitoring database and the existing rules. In a first phase, a study has to be conducted to select the correct and useful algorithms. Data can be clustered and classified, decision tree algorithms and pattern matching techniques should help us pander the necessary conclusions. When the data mining algorithms are developed and selected, they can be integrated into the Analysis module. This module will call the algorithms and combine the results to optimize the device choice algorithm.

\section{Contribution}

By focussing on the critical features and bottlenecks of clinical decision support systems, we want to improve the uptake of physicians and nurses. We want to provide them with a system fully integrated into their workflow. This means, enabling them to create clinical guidelines themselves and providing them with ways to automatically translate and execute them into applications. By using a pervasive model in combination with rules, algorithms are developed to decide which device is most suitable to display the information, given the current context. Monitoring all actions of the framework and the users will enable learning algorithms to improve the pervasive model and application specific rules.

\section{SPECIFIC ISSUES FOR THE DOCTORAL CONSORTIUM}

It would certainly benefit this doctoral research to address issues regarding the background to the research and also practicalities related to the planned work:

- As this research tries to find ways to integrate devices into the workflow of the ICU, it would be interesting to discuss how feasible it is to use the proposed technologies and if other existing technologies should be taken into consideration.

- How can we best trace the location of the user?

- How frequently does the system have to consult the context information? How can we reduce the overhead caused by using context? What techniques can be used to keep the overhead as low as possible?

- Which fallback methods can be used when for example context is not available? Should we apply general information and rules or are other options available?

\section{REFERENCES}

[1] D. Power and R. Sharda, "Decision Support Systems," in Springer Handbook of Automation, S. Y. Nof, Ed. Berlin, Heidelberg: Springer Berlin Heidelberg, 2009, ch. 87, pp. 1539-1548.

[2] J. Osheroff, J. Teich, B. Middleton, E. Steen, A. Wright, and D. Detmer, "A roadmap for national action on clinical decision support," JAMIA, vol. 14, no. 2, pp. 141-145, Mar. 2007.

[3] A. Garg, N. Adhikari, H. McDonald, P. Rosas-Arellano, P. Devereaux, J. Beyene, J. Sam, and B. Haynes, "Effects of computerized clinical decision support systems on practitioner performance and patient outcomes," JAMA, vol. 293, no. 10, pp. 1223-1238, Mar. 2005.

[4] A. Moxey, J. Robertson, D. Newby, I. Hains, M. Williamson, and S. Pearson, "Computerized clinical decision support for prescribing: Provision does not guarantee uptake," JAMIA, vol. 17, no. 1, pp. 25-33, Jan. 2010.

[5] C. Orwat, A. Graefe, and T. Faulwasser, "Towards pervasive computing in health care - a literature review," Social Science Research Network Working Paper Series, Sep. 2008.

[6] K. Kawamoto and D. Lobach, "Clinical decision support provided within physician order entry systems: A systematic review of features effective for changing clinician behavior," AMIA Annl Sym Proc, pp. 361-365, 2003.

[7] K. Colpaert, S. Vanbelleghem, C. Danneels, D. Benoit, K. Steurbaut, S. Van Hoecke, F. De Turck, and J. Decruyenaere, "Has information technology finally been adopted in Flemish Intensive Care Units?" $B M C$ Med Inform Decis Mak, vol. 10, no. 1, pp. 62+, Oct. 2010.

[8] K. Kawamoto, C. Houlihan, A. Balas, and D. Lobach, "Improving clinical practice using clinical decision support systems: a systematic review of trials to identify features critical to success," $B M J$, vol. 330 , no. 7494, pp. 765+, Apr. 2005.

[9] N. Bricon-Souf and C. Newman, "Context awareness in health care: A review," Int J Med Inform, vol. 76, no. 1, pp. 2-12, Jan. 2007.

[10] V. Herasevich, B. Pickering, Y. Dong, S. Peters, and O. Gajic, "Informatics infrastructure for syndrome surveillance, decision support, reporting, and modeling of critical illness." Mayo Clinic Proc, vol. 85, no. 3, pp. 247-254, Mar. 2010.

[11] D. Sittig, A. Wright, J. Osheroff, B. Middleton, J. Teich, J. Ash, E. Campbell, and D. Bates, "Grand challenges in clinical decision support." J Biomed Inform, vol. 41, no. 2, pp. 387-392, Apr. 2008.

[12] P. Soda, M. Pechenizkiy, F. Tortorella, and A. Tsymbal, "Knowledge discovery and Computer-Based decision support in biomedicine," Artif Intell Med, vol. 50, no. 1, pp. 1-2, Sep. 2010.

[13] J. Hewitt-Taylor, "Clinical guidelines and care protocols." Intensive Crit Care Nurs, vol. 20, no. 1, pp. 45-52, Feb. 2004.

[14] R. Shiffman, G. Michel, A. Essaihi, and E. Thornquist, "Bridging the guideline implementation gap: a systematic, document-centered approach to guideline implementation." JAMIA, vol. 11, no. 5, pp. 418 426, Sep. 2004.

[15] D. Wang, M. Peleg, S. Tu, A. Boxwala, R. Greenes, V. Patel, and E. Shortliffe, "Representation primitives, process models and patient data in computer-interpretable clinical practice guidelines: A literature review of guideline representation models," Int J Med Inform, vol. 68, no. 1-3, pp. 59-70, Dec. 2002.

[16] F. Buschmann, R. Meunier, H. Rohnert, P. Sommerlad, and M. Stal, Pattern-oriented Software Architecture - A System of Patterns. John Wiley \& Sons, Chichester, 1996.

[17] J. Rumbaugh, I. Jacobson, and G. Booch, Unified Modeling Language Reference Manual, The (2nd Edition). Pearson Higher Education, 2004.

[18] F. Ongenae, F. De Backere, K. Steurbaut, K. Colpaert, W. Kerckhove, J. Decruyenaere, and F. De Turck, "Towards computerizing intensive care sedation guidelines: design of a rule-based architecture for automated execution of clinical guidelines," BMC Med Inform Decis Mak, vol. 10 , no. 1, pp. 3+, Jan. 2010.

[19] F. De Backere, H. Moens, K. Steurbaut, F. De Turck, K. Colpaert, C. Danneels, and J. Decruyenaere, "Automated generation and deployment of clinical guidelines in the ICU," in Proc of CBMS. IEEE, Oct. 2010, pp. 197-202.

[20] F. De Backere, K. Steurbaut, K. Colpaert, J. Decruyenaere, and F. De Turck, "On the design of a management platform for antibiotic guidelines in the intensive care unit," in Proc of ICSEA. IEEE, Aug. 2010, pp. 406-411. 